\title{
Comparison and correlation between anterior and posterior elevation maps in normal and keratoconus patients
}

A.A.Hamed, H.M.Fayek, M.A.Abdrabbo and M.A.Riad

Ophthalmology, Dept., Faculty of Medicine, Benha Univ., Benha, Egypt

E-mail: nosabronze@gmail.com

\begin{abstract}
Background:Keratoconus $(\mathrm{KC})$ is a progressive disorder with conical deformity of the cornea. It is characterized by corneal thinning induces irregular astigmatism, myopia and protrusion resulting in mild to marked impairment in the quality of vision. Aim of work : Measuring posterior corneal elevation in normal and keratoconus corneas using pentacam to discriminate keratoconus from normal corneas and to compare and correlate anterior corneal elevation maps and posterior corneal elevation maps with severity of keratoconus. Methods: This study included 50 participants, $25 \mathrm{KC}$ patients and 25 healthy subjects. All cases were submitted to evaluation in the form of: history taking, visual acuity testing (UCVA, BCVA), slit lamp examination, corneal topography. Results: The comparison of keratometric and pachymetric readings showed significant differences between the study and control groups, central astigmatism (frontal and back), Kmax, KI, ISV and IVA show statistically significant difference between normal and keratoconic eyes. Posterior surface of the cornea shows statistically significant difference between normal and keratoconic eyes, In all keratoconus stages, the Posterior corneal elevation was significantly higher than the anterior corneal elevation by increasing in the keratoconus severity. Conclusion: Our data support the hypothesis that the posterior corneal surface contributes to the early topographical manifestation of keratoconus in keratoconus eyes. Although the diagnostic sensitivity was not explicitly evaluated in our study, giving more attention to posterior surface parameters may facilitate the early detection of keratoconus corneas.
\end{abstract}

Keywords: Keratoconus, Elevation maps, Pentacam.

\section{Introduction}

Keratoconus $(\mathrm{KC})$ is a progressive disorder with conical deformation of the cornea. It is characterized by corneal thinning that leads to irregular astigmatism, myopia and protrusion resulting in mild to marked impairment in the quality of vision .It is usually bilateral but asymmetrical; the apex of the cone is basically inferonasal. It is the most common primary ectasia and usually occurs in the second decade of life and affects both genders and all races. The estimated prevalence in the general population is 54 per 100,000 [1].

Keratoconus development involves a high degree of inter_and probably intralamellar displacement and slippage resulting in thinning of the central cornea and concomitant changes in curvature of the cornea. This slippage may be enhanced by a loss of cohesive forces and mechanical failure in regions where lamellae bifurcate [2].

Following the adventages of refractive surgerical methods such as photorefractive keratectomy (PRK) and laser-assisted in situ keratomileusis (LASIK), etc., there have been many reported cases of myopia progression due to rapid progression of central steepening postsurgery, classified as keratectasia and with a pathology similar to KC. Thus, specific diagnostic criteria regarding the development of $\mathrm{KC}$ is required, as it may contribute to not only the prevention of the progression to severe $\mathrm{KC}$, but also the general safety of refractive surgery[3].

Pentacam is an elevation-based diagnostic imaging system that is highly accurate in detecting $\mathrm{KC}$ and $\mathrm{KC}$ suspects [4].

\section{Patients and methods}

A cross sectional study includes 50 participant; 25 keratoconus patient and 25 healthy individuals.all are investigated by pentacam. They were selected from outpatient clinic of banha ophthalmology hospital. The study was conducted from septemper 2018 to septemper 2020 .

The study was approved by the ethical committee of Benha faculty of medicine. An informed written consent in Arabic language was obtained from all participants.

\subsection{Inclusion criteria}

Age more than 10 years, both genders, presence of normal lens and posterior chamber and clinical keratoconus. Soft contact lens users were included in the study after discontinuation of lens wear for at least 14 days.

\subsection{Exclusion criteria}

previous eye trauma, corneal or intraocular surgery, glaucoma, corneal scarring, severe eye dryness, current corneal infections, were using topical medications, and were pregnant or nursing. Eyes with pellucid marginal degeneration, inferior corneal thinning and ectasia were also excluded.

History was taking age, sex, onset of symptoms as itching, pain and blurring of vision and any previous ocular surgery.

\subsection{Ocular examination:}

1. Visual acuity: UCVA, BCVA using Snellen's chart for visual acuity 2. Slit lamp examination: for flisher ring, vogt stria ,munson's sign. 
2. Corneal topography: was performed to all participants using OCULUS Pentacam HR (Pentacam,wavelight, oculyzer II).

$\mathrm{KC}$ was diagnosed according to Amsler-Krumeich criteria and classification [5].

\section{Cases}

Case 1:

Female patient 18 years old presented with blurring of vision in left eye

$\mathrm{K} 1=46 \mathrm{D}, \mathrm{K} 2=47.8 \mathrm{D}$

Anterior corneal astigmatism $=1.8 \mathrm{D}$

Thinnest location $=487 \mu \mathrm{m}$

Anterior elevation at thinnest area $=11 \mu \mathrm{m}$

Posterior elevation at thinnest area $=25 \mu \mathrm{m}$

ISV $=43$

IVA $=0.52$

So this case (fig1) is stage I kc

\section{Case 2:}

Female patient 16 years old presented with diminution of vision Right eye;

$\mathrm{K} 1=44.5 \mathrm{D}, \mathrm{K} 2=52.9 \mathrm{D}$

Anterior corneal astigmatism $=8.3 \mathrm{D}$

Thinnest location $=486 \mu \mathrm{m}$

Anterior elevation at thinnest area $=24 \mu \mathrm{m}$

Posterior elevation at thinnest area $=41 \mu \mathrm{m}$

ISV $=79$

IVA $=0.72$
So this eye Pentacam (figure2) is stage II kc.

Case 3:

Female patient 25years old presented by diminution of vision

$\mathrm{K} 1=48.3 \mathrm{D}, \mathrm{K} 2=55.7 \mathrm{D}$

Anterior corneal astigmatism $=7.4 \mathrm{D}$

Thinnest location $=386 \mu \mathrm{m}$

Anterior elevation at thinnest area $=31 \mu \mathrm{m}$

Posterior elevation at thinnest area $=58 \mu \mathrm{m}$

ISV $=117$

IVA $=1.09$

$\mathrm{KC}$.

So this patient Pentacam (figure 3) is stage III

\section{Case 4 :}

Female patient 34years old presented by progressive deterioration of vision

$\mathrm{K} 1=55.7 \mathrm{D}, \mathrm{K} 2=65.2 \mathrm{D}$

Anterior corneal astigmatism $=9.5 \mathrm{D}$

Thinnest location $=365 \mu \mathrm{m}$

Anterior elevation at thinnest area $=69 \mu \mathrm{m}$

Posterior elevation at thinnest area $=126 \mu \mathrm{m}$

ISV $=176$

IVA $=1.48$ $\mathrm{KC}$.

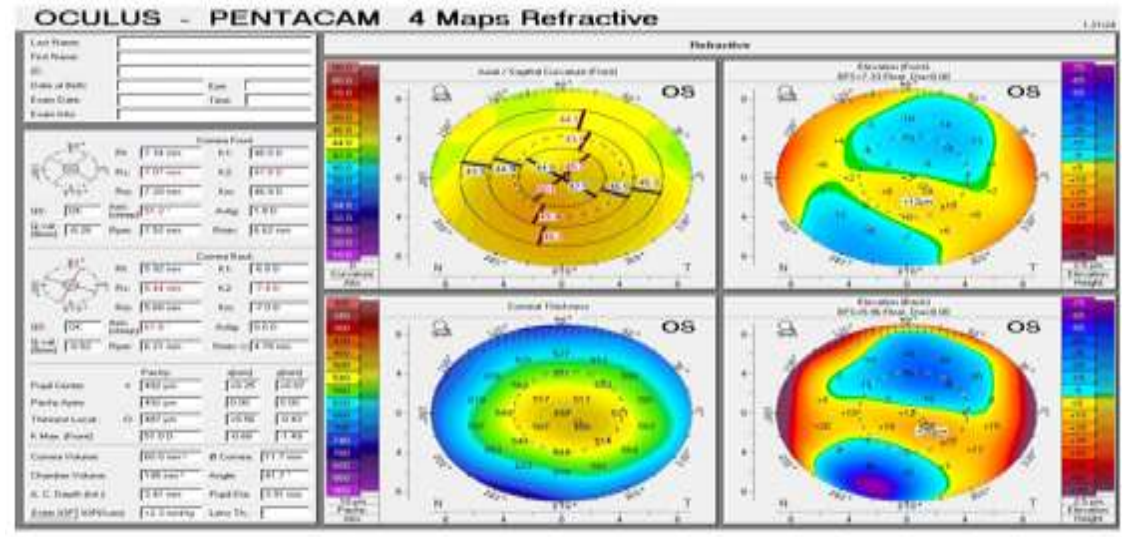

Fig. (1) stage I KC in left eye .

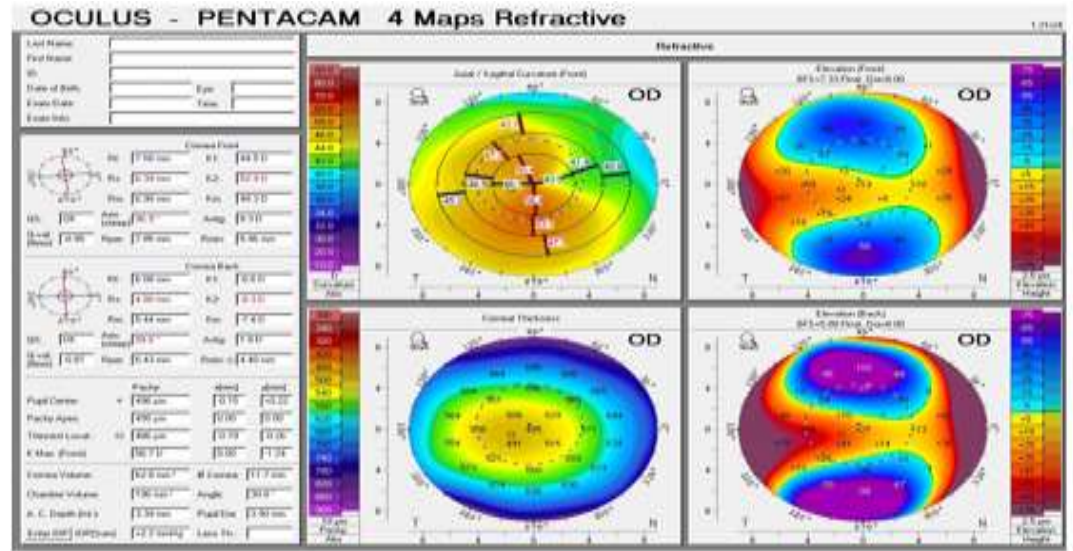

Fig. (2) stage II KC in right eye. 


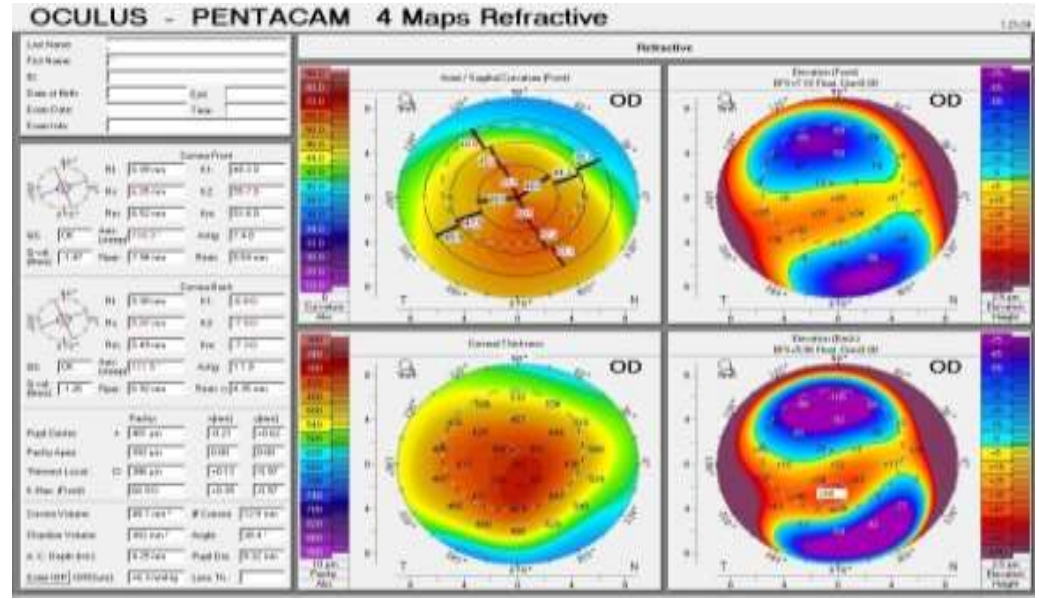

Fig. (3) stage III KC in right eye.

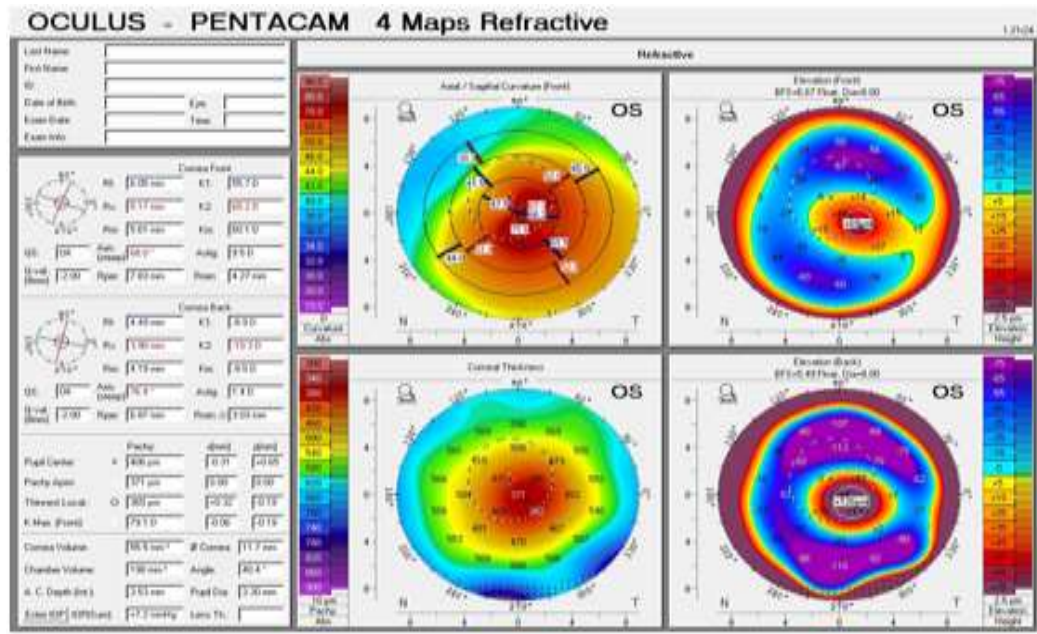

Fig. (4) stage IV KC in left eye.

\subsection{Statistical methods}

Data management and statistical analysis were done using SPSS vs.25. (IBM, Armonk, New York, United States). Numerical data were summarized as means and standard deviations or medians and ranges. Categorical data were summarized as numbers and percentages. Comparisons between both groups were done using independent t-test or MannWhitney $U$ test for normally and non-normally distributed numerical data, respectively. Categorical data were compared using the Chi-square test. All P values were two-sided. $\mathrm{P}$ values less than 0.05 were considered significant.

\section{Results}

This study consisted of two groups; Group I: 25 patients with keratoconus (49 eyes). and Group II: 25 individuals with normal eyes (25 eyes). There were no significant differences between both groups as regard age and gender. $\mathrm{P}$ values were 0.574 and 1.0, respectively, table (1).

The Independent t-test was used for age. Chisquare test was used for gender
There was no significant difference between both groups regarding OD/OS status; P-value was 0.806 . Mean minimal pachymetry was significantly lower in cases (448) than controls (544); the P-value was $<0.001$. Regarding mean $\mathrm{K} 1$ and $\mathrm{K} 2$, they were significantly higher in group I (48.5 \& 51.6, respectively) than group II $(42.7 \quad \& \quad 44$, respectively); $\mathrm{P}$ values were $<0.001$ for each. Mean $\mathrm{Km}$ was significantly higher in group I (49.6) than group II (43.3); P-value was <0.001. Median anterior and posterior astigmatism were significantly higher in group I ( $3.3 \& 0.7$, respectively) than group II $(-0.4 \&$ 0.3 , respectively); $\mathrm{P}$ values were $<0.001$ for each. Mean anterior and posterior elevation were significantly higher in group I (27\&55, respectively) than group II (4\&6, respectively); $\mathrm{P}$ values were $<0.001$ for each. ISV was significantly higher in group I (83) than group II (20); the P-value was $<0.001$. IVA was significantly higher in group I (0.79) than group II (0.15); P-value was $<0.001$. Also, KI was significantly higher in group I (1.21) than group II (1.02); P-value was <0.001, table (2) \& figure (5). 
The most frequent keratoconus stage was stage II (49\%), followed by stage III $(22.4 \%)$, then stage I $(20.4 \%)$, and stage IV (8.2\%), figure (6)

Anterior and posterior elevation in different keratoconus stages and controls.In controls, there was no significant difference between anterior and posterior elevation; P-value was 0.110 . In all keratoconus stages, the Posterior corneal elevation was significantly higher than the anterior corneal elevation. In stage I, the posterior elevation was 27 while the anterior elevation was 11 , the P-value was $<0.001$. In stage II, the posterior elevation was 52 while the anterior elevation was 26 , the P-value was $<0.001$. In stage III, the posterior elevation was 35 while the anterior elevation was 67 , the P-value was $<0.001$. In stage IV, the posterior elevation was 109 while the anterior elevation was 56 , the P-value was 0.013 (figure 7)

Table (1) Demographic characteristics in both groups.

\begin{tabular}{|c|c|c|c|c|}
\hline & & $\begin{array}{c}\text { Cases } \\
(\mathrm{n}=25 \text { patients })\end{array}$ & $\begin{array}{c}\text { Controls } \\
(\mathrm{n}=\mathbf{2 5} \text { patients })\end{array}$ & P value \\
\hline Age & Mean \pm SD & $26 \pm 6$ & $27 \pm 7$ & 0.574 \\
\hline \multirow[t]{2}{*}{ Gender } & Males $\quad n(\%)$ & $10(40)$ & $10(40.0)$ & 1.0 \\
\hline & Females $n(\%)$ & $15(60)$ & $15(60.0)$ & \\
\hline
\end{tabular}

Table (2) Pentacam findings in both groups

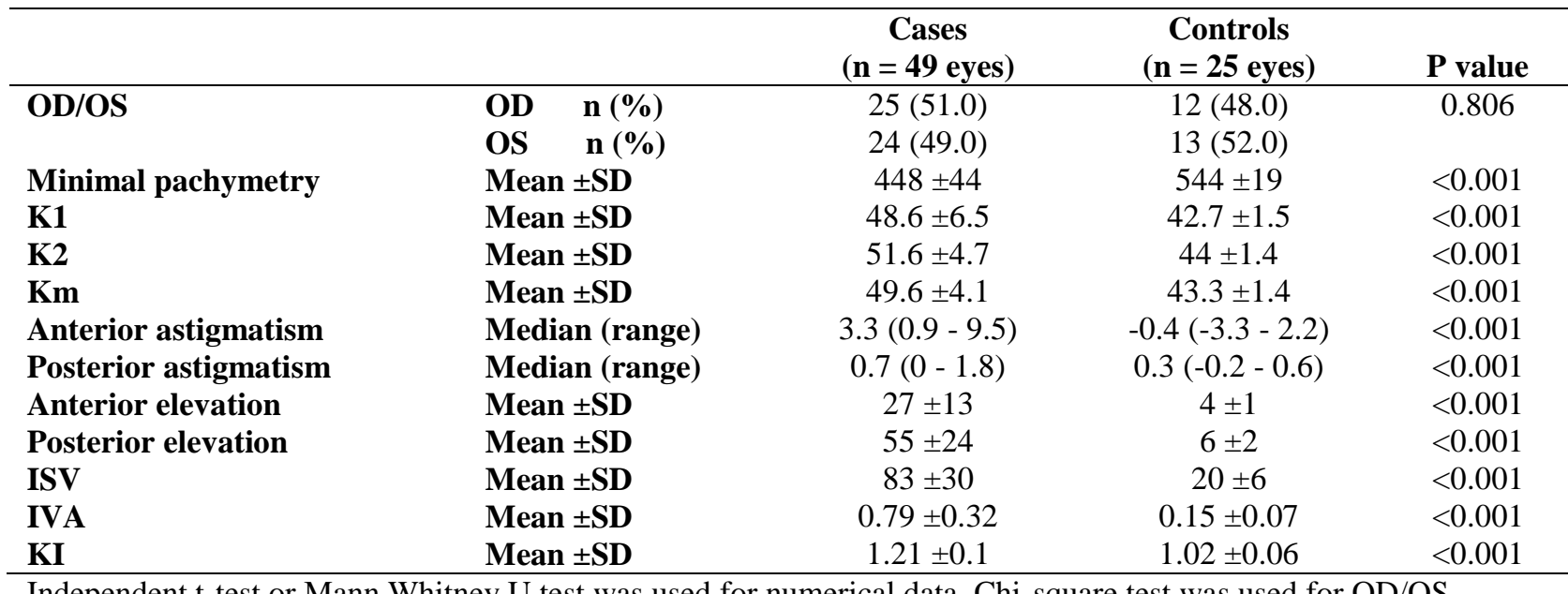

Independent t-test or Mann Whitney U test was used for numerical data. Chi-square test was used for OD/OS

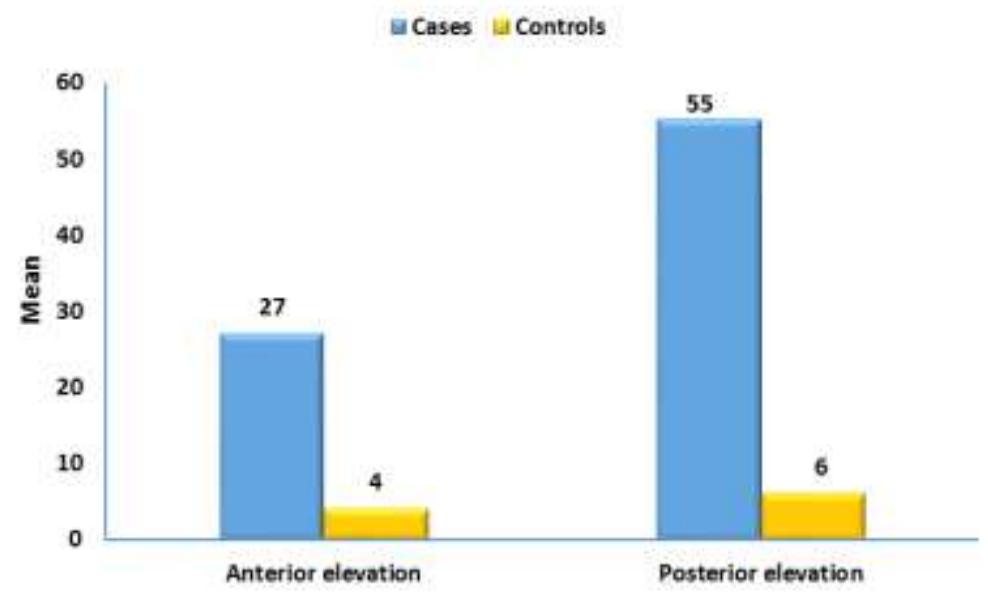

Fig. (5) Anterior and posterior elevation in both groups. 
Stage I $\square$ Stage II $\square$ Stage III $\square$ Stage IV

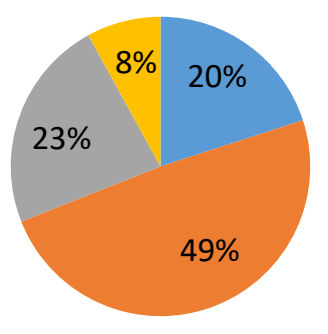

Fig. (6) Distribution of keratoconus stages in group I.

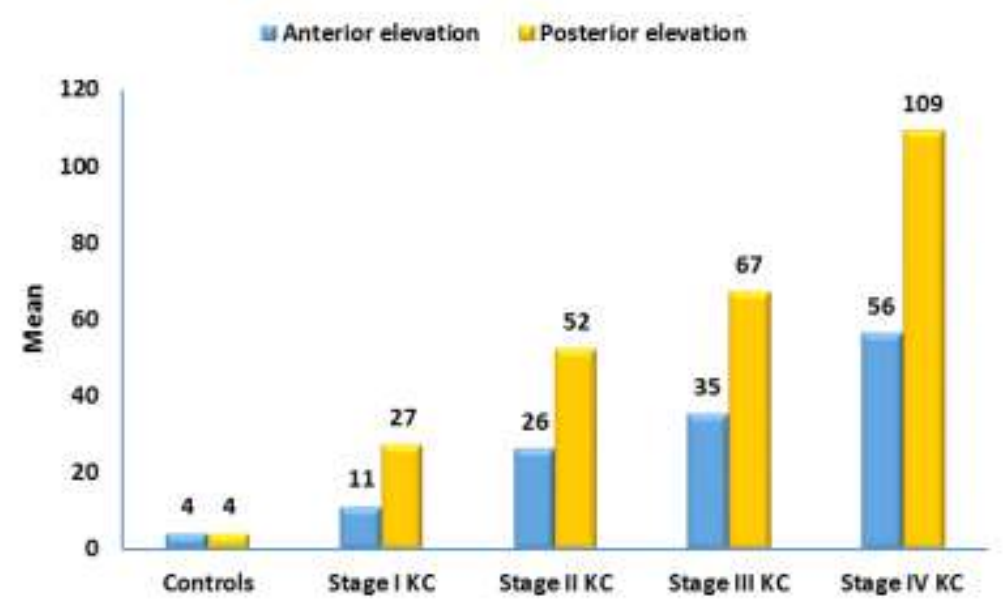

Fig. (7) Anterior and posterior elevation in different keratoconus stages and controls.

\section{Discussion}

Keratoconus diagnosis based on curvature topography and pachymetric measurement is often difficult. More recent studies, based on the elevation data obtained by Pentacam measurements, suggest that posterior surface corneal elevation is an early and sensitive marker of keratoconus, Another advantage of the posterior corneal surface analysing is that this map is not affected by tear-film irregularities.

The study evaluated the association between corneal anterior elevation and posterior elevation with severity of keratoconus. The result of this study showed significant positive correlation between keratoconus severity and corneal elevations (anterior and posterior elevation).the analyses showed that anterior and posterior elevations have the best reference of keratoconus staging, indicating that corneal elevation from Pentacam have the best predictive accuracy for keratoconus grading.

In this study, $20.4 \%$ of eyes of patients are stage $1,49 \%$ of eyes of patients is stage II. And $22.4 \%$ of eyes of patients are stage III and $8.2 \%$ of eyes of patients are stage IV, so stage II is the most common stage in this study. While Kamiya et al, [6] showed that $28.6 \%$ of eyes are stage I ,27\% of eyes are stage II, $15.1 \%$ of eyes are stage III and $29.3 \%$ of eyes are stage IV with stage I is the commonest. And Naderan et al, [7] Showed that $47.3 \%$ of eyes are stage I , $35.6 \%$ of eyes are stage II , $11 \%$ of eyes are stage III ,and $6.1 \%$ of eyes are stage IV with also stage I is the commonest .

Other studies agreed with this study in results such as:Tomidokoro et al, [8] reported that all refractive components showed statistically significant correlations between the anterior and posterior surfaces $(\mathrm{P}<0.0001)$.so not only the anterior but also the posterior corneal curvature is affected in keratoconus. These changes are noticed from the early stage of keratoconus.

de Sanctis et al, [9] reported that : Mean posterior corneal elevation was statistically higher in keratoconus $(100.7 \pm 49.2 \mu \mathrm{m} \quad \mathrm{p}<0.001)$ than normal corneas $(19.8 \pm 6.37 \mu \mathrm{m})$. . Receiver operating characteristic (ROC) curve analyses showed high general predictive accuracy of posterior elevation for keratoconus (area under the curve 0.99). Optimal cutoff points were $35 \mu \mathrm{m}$ for keratoconus. These values had been associated with sensitivity and specificity of $97.3 \%$ and $96.9 \%$, respectively, for keratoconus .Similar cut-off points were obtained with logistic regression analysis $(38 \mu \mathrm{m}$ for keratoconus).so posterior corneal elevation effectively distinguish keratoconus from normal corneas. 
Miha'ltz et al, [10] All parameters were significantly different in the keratoconus group compared to the normal control group. ROC analyses showed the best predictive accuracy for posterior and anterior elevation (area under the curve, 0.97 and $0.96)$ followed by minimal and central pachymetry (0.89 and 0.88). The optimal cut-off point for posterior elevation was $15.5 \mu \mathrm{m}$ to distinguish keratoconus corneas from normal. Logistic regression analysis showed the best fit to the model data completed with the elevation data of the Pentacam. Confirmatory factor analysis demonstrate that a 3-factor model satisfactorily showing minimal pachymetry (-0.99), anterior elevation (0.98), and keratometry (0.95) as the most representative clinical variants of the disease. So, posterior and anterior elevation, pachymetric, and keratometric parameters measured by the Pentacam can effectively distinguish keratoconus from normal corneas which serves as a useful diagnostic tool for staging disease.

Ishii et al, [11] found that there was a significant positive correlation between elevation differences and keratoconus severity index in both anterior and posterior surfaces (Pearson correlation coefficient, $r=0.66 ; P<0.001 ; r=0.74 ; P<0.001)$. the incidences of greater elevation differences in the anterior and posterior corneal surfaces were higher in Amsler-Krumeich classification (1-way analysis of variance, $P=0.040 ; P<0.001)$. the lower staging cases in the Amsler-Krumeich classification had a larger area under the ROC in the posterior elevation compared to the anterior elevation, indicating a greater diagnostic value of the posterior elevation measurement.

Kamiya et al, [6] showed that posterior (0.980) and anterior (0.977) elevation differences showed the highest AUROCs, followed by minimal pachymetry (0.923), average keratometry (0.914), anterior elevation (0.909), posterior elevation (0.898), central pachymetry (0.889), corneal thickness spatial profile $2 \mathrm{~mm}(0.835)$ and cylinder (0.796). The differences in AUROC curves between measurements of anterior and posterior elevation difference and other diagnostic parameters tended to be greater in the early stages of keratoconus. So that Anterior and posterior corneal surface elevation data obtained through enhanced ectasia display, effectively distinguish keratoconus from normal corneas. Elevation difference measurements may provide useful information to improve the diagnostic accuracy of keratoconus, especially in the early stage of the disease.

Medghalchi et al, [12] found that There was a strong positive correlation between keratoconus severity and corneal elevations (anterior and posterior elevation as measured with both conventional and enhanced best-fit spheres) and also between keratoconus severity and corneal elevation differences $(\mathrm{P}<0.001$ and $\mathrm{r}>0.625$ for all).
Maximum keratometry (Kmax), mean keratometry (Kmean), and all corneal elevations and difference elevations were highly correlated $(\mathrm{P}<0.001$ and $\mathrm{r}>$ 0.840 for all. ROC curve analyses showed that the anterior and posterior elevations have the best predictive accuracy for staging severity of keratoconus. So the evaluation of corneal elevation data obtained from Scheimpflug corneal imaging is useful for grading the severity of keratoconus.

\section{Conclusion}

Our data support the hypothesis that the anterior and posterior corneal surface contributes to discriminate beween normal and keratoconus corneas, and the early topographical manifestation in keratoconus eyes. Although the diagnostic sensitivity was not explicitly evaluated in our study, giving more attention to posterior surface parameters may facilitate the early detection of keratoconus corneas.

\section{Sources of funding}

This research did not receive any specific grant from funding agencies in the public, commercial, or not-for-profit sectors.

\section{Author contribution}

Authors contributed equally in the study.

\section{Conflicts of interest}

No conflicts of interest

\section{References}

[1] Romero-Jiménez, Miguel, Jacinto Santodomingo-Rubido, and James S. Wolffsohn. "Keratoconus: a review." Contact Lens and Anterior Eye.vol. 33, pp. 157-166,2010.

[2] Meek, M.Keith, J.Stephen Tuft, Yifei Huang, S.Paulvinder Gill, Sally Hayes, H. Richard Newton, and J.Anthony Bron. "Changes in collagen orientation and distribution in keratoconus corneas." Investigative ophthalmology \& visual science.vol. 46,pp.1948-1956. ,2005.

[3] Itoi, Motohiro, Koji Kitazawa, Isao Yokota, Koichi Wakimasu, Yuko Cho, Yo Nakamura, Osamu Hieda, Satoshi Teramukai, Shigeru Kinoshita, and Chie Sotozono. "Anterior and posterior ratio of corneal surface areas: A novel index for detecting early stage keratoconus." PloS one.vol. 15,pp. e0231074,2020.

[4] Mazen M Sinjap "corneal topography in clinical practice (Pentacam system) basic \& clinical interpretation " chapter.vol. 4,pp. 40-46,2020.

[5] M.W.Belin, and J.K.Duncan. "Keratoconus: the ABCD grading system." Klinische Monatsblätter für Augenheilkunde.vol. 233,pp. 701-707,2016.

[6] Kamiya, Kazutaka, Rie Ishii, Kimiya Shimizu, and Akihito Igarashi. "Evaluation 
of corneal elevation, pachymetry and keratometry in keratoconic eyes with respect to the stage of Amsler-Krumeich classification." British Journal of Ophthalmology.vol. 98,pp.459-463. ,2014.

[7] Naderan, Mohammad, Saeed Shoar, Mohammad, A.Kamaleddin, T.Mohammad Rajabi, Morteza Naderan, and Marjan Khodadadi. "Keratoconus clinical findings according to different classifications." Cornea.vol. 34,pp. 1005$1011,2015$.

[8] Tomidokoro, Atsuo, Tetsuro Oshika, Shiro Amano, Shiro Higaki, Naoyuki Maeda, and Kazunori Miyata. "Changes in anterior and posterior corneal curvatures in keratoconus." Ophthalmology.vol. 107,pp. 1328-1332,2000.

[9] De Sanctis, Ugo, Carlotta Loiacono, Lorenzo Richiardi, Davide Turco, Bernardo Mutani, and Federico M. Grignolo. "Sensitivity and specificity of posterior corneal elevation measured by Pentacam in discriminating keratoconus/subclinical keratoconus." Ophthalmology.vol. 115,pp. 1534-1539,2008.

[10] Miháltz, Kata, Illés Kovács, Ágnes Takács, and Zoltán Z. Nagy. "Evaluation of keratometric, pachymetric, and elevation parameters of keratoconic corneas with pentacam." Cornea.vol. 28 ,pp.976980,2009.

[11] Ishii, Rie, Kazutaka Kamiya, Akihito Igarashi, Kimiya Shimizu, Yoshikazu Utsumi, and Takashi Kumanomido. "Correlation of corneal elevation with severity of keratoconus by means of anterior and posterior topographic analysis." Cornea.vol.31,pp. 253-258,2012.

[12] Medghalchi, Abdolreza, Reza Soltani Moghadam, Mitra Akbari, Yousef Alizadeh, Soheil Soltanipour, Heidar Veisi, and Yaser Khakpour. "Correlation of corneal elevations measured by Scheimpflug corneal imaging with severity of keratoconus." Journal of current ophthalmology.vol. 31,pp. 377-381,2019. 